\title{
ALBI grade in dialysis patients with hepatocellular carcinoma: prognostic impact and staging strategy
}

\author{
Shu-Yein Ho ${ }^{1,2,3}$, Po-Hong Liu ${ }^{3,4}$, Chia-Yang Hsu ${ }^{3,5}$, Chih-Chieh Ko ${ }^{3,6}$, Yi-Hsiang Huang ${ }^{3,6,7}$, \\ Chien-Wei Su ${ }^{3,6}$, Cheng-Yuan Hsia ${ }^{3,8}$, Rheun-Chuan Lee ${ }^{3,9}$, Ming-Chih Hou ${ }^{3,6}$, Teh-Ia Huo ${ }^{2,3,10}$ \\ ${ }^{1}$ Division of Gastroenterology and Hepatology, Min-Sheng General Hospital, Taoyuan, Taiwan; ${ }^{2}$ Department of Medical Research, Taipei Veterans \\ General Hospital, Taipei, Taiwan; ${ }^{3}$ Faculty of Medicine, National Yang-Ming University School of Medicine, Taipei, Taiwan; ${ }^{4}$ Department of \\ Internal Medicine, University of Texas Southwestern Medical Center, Dallas, Texas, USA; ${ }^{5}$ Division of Gastroenterology and Hepatology, University \\ of Michigan, Ann Arbor, MI, USA; ${ }^{6}$ Department of Medicine, Taipei Veterans General Hospital, Taipei, Taiwan; ${ }^{7}$ Institute of Clinical Medicine, \\ National Yang-Ming University School of Medicine, Taipei, Taiwan; ${ }^{8}$ Department of Surgery, Taipei Veterans General Hospital, Taipei, Taiwan; \\ ${ }^{9}$ Department of Radiology, Taipei Veterans General Hospital, Taipei, Taiwan; ${ }^{10}$ Institute of Pharmacology, National Yang-Ming University School of \\ Medicine, Taipei, Taiwan \\ Contributions: (I) Conception and design: YH Huang, CC Ko, RC Lee, MC Hou; (II) Administrative support: TI Huo; (III) Provision of study \\ materials or patients: TI Huo; (IV) Collection and assembly of data: SY Ho; (V) Data analysis and interpretation: CY Hsu, PH Liu, CY Hsia, CW \\ $\mathrm{Su}$; (VI) Manuscript writing: All authors; (VII) Final approval of manuscript: All authors. \\ Correspondence to: Teh-Ia Huo, MD. Professor of Medicine, Department of Medical Research, Taipei Veterans General Hospital, No. 201, Sec. 2, \\ Shipai Rd., Taipei, Taiwan. Email: tihuo@vghtpe.gov.tw.
}

Background: Patients with hepatocellular carcinoma (HCC) may develop end-stage renal disease and receive dialysis, but the impact of dialysis on the prognosis is unclear. This study aimed to evaluate the outcome of dialysis HCC patients and the prognostic role of albumin-bilirubin (ALBI) grade in these patients.

Methods: Among the consecutive 3,794 HCC patients between 2002-2017, 43 patients undergoing dialysis, and 129 age, sex-matched controls were analyzed. Multivariate Cox hazards model was used to identify independent prognostic predictors.

Results: Dialysis patients had decreased overall survival when compared with non-dialysis patients $(\mathrm{n}=3,751)$ and matched controls ( $\mathrm{n}=129$; each $\mathrm{P}=0.004)$. Patients with ALBI grade 1 had the best survival in the pooled cohort of dialysis and matched controls $(\mathrm{n}=172)$. In the Cox model, total tumor volume $>33 \mathrm{~cm}^{3}$ [hazard ratio (HR): 6.763, $\mathrm{P}<0.001$ ], presence of ascites (HR: 6.168, $\mathrm{P}<0.001$ ), dialysis duration less than 24 months (HR: 3.144, $\mathrm{P}=0.006)$, diabetes-related dialysis (HR: 9.366, $\mathrm{P}=0.001)$ and non-curative treatments (HR: 9.220, $\mathrm{P}<0.001)$ were poor prognosis factors associated with increase mortality among dialysis patients. Of the 9 currently-used HCC staging systems, the CLIP score was the optimal cancer staging for dialysis patients.

Conclusions: Patients receiving dialysis had decreased overall survival compared with non-dialysis patients. Longer duration of dialysis, non-diabetes related dialysis, absence of ascites, and curative treatments were associated with improved survival in these patients. The ALBI grade is a feasible prognostic model to evaluate liver functional reserve, and the CLIP model is the best staging system for dialysis patients with HCC.

Keywords: Dialysis; hepatocellular carcinoma (HCC); albumin-bilirubin grade (ALBI grade)

Submitted Aug 14, 2020. Accepted for publication Dec 14, 2020.

doi: 10.21037/jgo-20-332

View this article at: http://dx.doi.org/10.21037/jgo-20-332 


\section{Introduction}

Hepatocellular carcinoma (HCC) is the most common type of liver cancer and the fourth leading cause of cancerrelated death globally with increasing incidence (1). The major risk factors for HCC are chronic viral hepatitis B and $\mathrm{C}$ (HBV, HCV), alcoholism and metabolic syndrome $(2,3)$. HCC patients often coexist with liver cirrhosis which may predispose to renal insufficiency because of vasodilatation of splanchnic circulation, decreased effective blood volume, activation of vasoconstrictors and renal hypoperfusion (4).

Renal dysfunction occurs in about $25 \%$ of HCC patients (5). Previous studies showed that renal insufficiency was a negative prognostic predictor in HCC patients receiving surgical resection and transarterial chemoembolization $(6,7)$. Of these patients, some could develop end-stage renal disease (ESRD) and require dialysis. Dialysis patients had an increased risk of uremia-related complications including cardiovascular events, chronic viral hepatitis, hypotension, and various microbial infections (8-10). Notably, 70-90\% of HCC patients had renal dysfunction due to underlying chronic liver disease or cirrhosis, and various degrees of liver functional reserve are usually present in dialysis patients with HCC upon diagnosis.

The management of HCC depends on tumor burden and the severity of liver functional reserve. The ChildTurcotte-Pugh (CTP) score has been proposed to assess the degree of liver dysfunction. However, the CTP has its limitation because some parameters are based on arbitrarily defined cut-offs. Moreover, the interpretation of hepatic encephalopathy and ascites are subjective, and serum albumin and ascites are often inter-related. Alternatively, the model for end-stage liver disease (MELD) is an objective method, but its role is primarily for cirrhotic patients awaiting liver transplantation (11). Recently, the albumin-bilirubin (ALBI) grade, consisting of only serum albumin and bilirubin, was reported a simple and objective method to assess hepatic dysfunction. The feasibility of ALBI grade in HCC has been validated by several groups (12-15). However, the prognostic role of ALBI grade in HCC patients receiving dialysis has not been evaluated.

Multiple staging systems including Barcelona Clinic Liver Cancer (BCLC), Cancer of the Liver Italian Program (CLIP), Chinese University Prognostic Index (CUPI), Hong Kong Liver Cancer (HKLC), Japan Integrated Staging (JIS) system, Taipei Integrated Scoring (TIS) system, Tumor-Node-Metastasis (TNM) system, Okuda system, and Tokyo system, have been proposed for HCC
$(16,17)$. The prognostic accuracy of these staging systems in HCC patients receiving dialysis has not yet been determined. We aimed to assess the prognostic role of ALBI grade in dialysis HCC patients and determine the optimal staging system for this special patient group.

We present the study in accordance with the REMARK reporting checklist (available at http://dx.doi.org/10.21037/ jgo-20-332).

\section{Methods}

\section{Patients}

During the 16-year period between 2002 to 2017, 3,794 HCC patients in our hospital were prospectively enrolled and retrospectively analyzed. Patients receiving maintenance dialysis were identified at the time of diagnosis based on medical charts. The baseline characteristics, including the underlying of liver disease, the degree of liver function reserve, serum biochemistries, tumor burden (size, number, vascular invasion, and distant metastasis), performance status, cancer stage, treatment, etiology of chronic kidney disease, duration, and forms of dialysis, were recorded upon diagnosis. Patients receiving dialysis for less than 3 months were excluded from this study. For analysis, dialysis patients were matched with patients without dialysis based on age and sex in a 1:3 ratio to specifically evaluate their outcome. The survival of patients was inspected every 3-4 months until death or dropout from the follow-up program.

\section{Ethical statement}

The study was approved by the Institutional Review Board of Taipei Veterans General Hospital (No. 2018-03-004CC) and complies with the standards of the Declaration of Helsinki (as revised in 2013) and current ethical guidelines. Informed consent was obtained before treatment.

\section{Diagnosis and definition}

The diagnosis of HCC was based on typical image finding according to current European Association for the Study of Liver (EASL) and American Association for the Study of Liver Diseases (AASLD) practice guidelines $(1,2)$. Tumor invasion to branch or main portal vein, or inferior vena cava on CT scan or MRI was denoted as vascular invasion. Distant metastasis, such as lung, lymph node and bone, was confirmed by CT, MRI, or bone scan (18). Performance 
status was assessed by using the Eastern Cooperative Oncology Group (ECOG) performance scale (19). The calculation of total tumor volume (TTV) was previously described (20). The equation of model for end-stage liver disease (MELD) score was provided in our previous study (21). The ALBI score was calculated according to the following equation $=0.66 \times \log _{10}$ bilirubin $(\mu \mathrm{mol} / \mathrm{L})-$ $0.085 \times$ albumin $(\mathrm{g} / \mathrm{L})$. ALBI grades were classified into three groups: ALBI grade 1 (score $\leq-2.60$ ), ALBI grade 2 (score $>-2.60$ and $\leq-1.39$ ) and ALBI grade 3 (score $>-1.39$ ) $(12,14)$. The equation of estimated glomerular filtration rate (eGFR) was calculated based on the modification of diet in renal disease (MDRD) formula (22). The underlying etiology of chronic kidney disease was classified as diabetes nephropathy and non-diabetes cause such as hypertension, renal artery stenosis, and chronic glomerulonephritis. The forms of dialysis included hemodialysis and peritoneal dialysis.

\section{Treatments}

Patients were discussed in the multidisciplinary board conference of Taipei Veterans General Hospital for diagnosis and treatment guidance. The risks and benefits of therapeutic information were explained to each patient. Shared decisions were made by the patients and physicians after individual counseling. Informed consent was obtained before treatment. The procedure of surgical resection, radiofrequency ablation (RFA), and transarterial chemoembolization (TACE) has been prescribed in our previous study. (23). Surgical resection, local ablation therapy, and liver transplantation were collectively defined as curative treatments, and TACE, chemo- or targeted therapy, and other treatments were classified as non-curative treatments.

\section{Statistic analysis}

All statistical analyses were performed by using IBM SPSS Statistics for Windows, version 21.0 (IBM Corp., Armonk, NY, USA). The Kaplan-Meier analysis with the log-rank test was used to evaluate in univariate survival analysis. Multivariate Cox proportional hazards model was used to identify the independent prognostic predictors and adjusted hazard ratio (HR). Corrected Akaike information criterion (AICc) was obtained to reveal how staging system correlated with patient survival. Homogeneity was measured by $\chi^{2}$ test to evaluate the differences in survival among patients in the same stage within each system. A P value of less than 0.05 was considered statistically significant.

\section{Results}

\section{Baseline characteristics}

Table 1 shows the comparison of baseline information of all study patients, matched control, and dialysis patients. The mean age of dialysis patients was 62 years, and the majority $(65 \%)$ of patients were male. HCV (30\%) and HBV (28\%) were the main cause of chronic liver disease in dialysis patients. Dialysis patients had higher percentage of dual HBV and HCV (9\%) infection compared with other two cohorts. The dialysis group had a significant lower albumin level ( $\mathrm{P}=0.028$ and 0.042 , respectively), higher bilirubin level $(\mathrm{P}=0.005$ and 0.009 , respectively), higher creatinine level $(\mathrm{P}<0.001)$, lower eGFR level $(\mathrm{P}<0.001)$, lower serum $\alpha$-fetoprotein (AFP) level $(\mathrm{P}<0.001)$, larger TTV $(\mathrm{P}<0.001)$ compared with other two cohorts. Dialysis patients had a worse performance status compared with non-dialysis and matched controls ( $\mathrm{P}=0.017$ and 0.018 , respectively). Dialysis patients had higher MELD scores when compared with other two cohorts (all $\mathrm{P}<0.001)$. The presence of ascites was also higher in dialysis patients compared with other two cohorts $(\mathrm{P}<0.001$ and $\mathrm{P}=0.008$ respectively). Otherwise, there was no significant difference in vascular invasion, distant metastasis, treatment, BCLC staging and CLIP score between dialysis patients and the other two cohorts.

\section{Comparison of overall survival between dialysis and non- dialysis patients}

The median survival of dialysis patients, non-dialysis patients, and matched controls were 16 [95\% confidence interval (CI): 8.6-23.4] months, 28 (95\% CI: 25.6-30.3) months, and 38 (95\% CI: 25-51) months, respectively. Dialysis HCC patients had increased risk of mortality compared with non-dialysis patients $(\mathrm{P}=0.004$, Figure $1 A)$, and matched controls $(\mathrm{P}=0.004$, Figure $1 B)$. The survival probabilities at 1,3 and 5 years were $55 \%, 33 \%$ and $14 \%$ in dialysis patients, $66 \%, 45 \%, 34 \%$ in non-dialysis patients, and $68 \%, 51 \%, 36 \%$ in matched controls, respectively. Among patients receiving dialysis, 36 (84\%) patients died during the study period. The cause of death was classified as hepatic-related and non-hepatic related. Eighteen (50\%) of patients died of hepatic cause (tumor progression, hepatic failure) and others died of non-hepatic causes (sepsis and others). 
Table 1 Comparison of baseline characteristics of dialysis $(n=43)$, non-dialysis $(n=3,751)$ and age, sex-matched controls $(n=129)$ HCC patients

\begin{tabular}{|c|c|c|c|c|c|}
\hline Variables & Dialysis patients & Non-dialysis patients & $\mathrm{P}$ & Matched-control & $\mathrm{P}$ \\
\hline Age (years, mean \pm SD) & $62 \pm 12$ & $65 \pm 13$ & 0.211 & $62 \pm 12$ & 0.980 \\
\hline Male/female, n (\%) & $28(65) / 15(35)$ & 2,867 (76)/884 (24) & 0.103 & $84(65) / 45(35)$ & 1.000 \\
\hline \multicolumn{2}{|c|}{ Etiologies of liver disease, n (\%) } & & 0.068 & & 0.100 \\
\hline HCV, n (\%) & $13(30)$ & $811(22)$ & & $33(26)$ & \\
\hline HBV + HCV, n (\%) & $4(9)$ & $131(4)$ & & $3(2)$ & \\
\hline Others, n (\%) & $14(33)$ & $1,308(34)$ & & $37(29)$ & \\
\hline \multicolumn{6}{|c|}{ Laboratory values (mean \pm SD) } \\
\hline ALT (IU/L) & $68 \pm 104$ & $70 \pm 91$ & 0.892 & $76 \pm 108$ & 0.687 \\
\hline Creatinine (mg/dL) & $8.3 \pm 2.5$ & $1.1 \pm 0.6$ & $<0.001$ & $1.1 \pm 0.6$ & $<0.001$ \\
\hline Sodium (mmol/L) & $137 \pm 4$ & $138 \pm 4$ & 0.016 & $139 \pm 4$ & 0.022 \\
\hline INR of PT & $1.1 \pm 0.2$ & $1.1 \pm 0.3$ & 0.737 & $1.1 \pm 0.2$ & 0.909 \\
\hline Platelet (1,000 ㅆL/L) & $154 \pm 99$ & $170 \pm 96$ & 0.261 & $170 \pm 106$ & 0.386 \\
\hline $\begin{array}{l}\text { AFP }(\mathrm{ng} / \mathrm{mL}) \\
\text { median }[\mathrm{IQR}]\end{array}$ & $10[4-139]$ & $45[8-826]$ & $<0.001$ & $51[9-764]$ & $<0.001$ \\
\hline eGFR $\left(\mathrm{mL} / \mathrm{min} / 1.73 \mathrm{~m}^{2}\right)$ & $6.9 \pm 3.0$ & $76 \pm 30$ & $<0.001$ & $77 \pm 32$ & $<0.001$ \\
\hline $\begin{array}{l}\text { Vascular invasion or } \\
\text { metastasis, } \mathrm{n}(\%)\end{array}$ & $10(23)$ & $1,028(28)$ & 0.531 & $32(25)$ & 0.838 \\
\hline Ascites, $\mathrm{n}(\%)$ & $19(44)$ & $842(23)$ & 0.001 & $30(23)$ & 0.008 \\
\hline $\mathrm{DM}, \mathrm{n}(\%)$ & $13(30)$ & $959(26)$ & 0.486 & $31(24)$ & 0.420 \\
\hline CTP class (A/B/C), n (\%) & $27 / 12 / 4(63 / 28 / 9)$ & $2,760 / 819 / 172(74 / 21 / 5)$ & 0.110 & $92 / 34 / 3(71 / 27 / 3)$ & 0.121 \\
\hline CTP score (mean \pm SD) & $6.6 \pm 1.8$ & $6.0 \pm 1.5$ & 0.023 & $6.1 \pm 1.5$ & 0.068 \\
\hline $\begin{array}{l}\text { MELD (<8/8-14/ } \\
14-20 />20), n(\%)\end{array}$ & 0/0/11/23 (0/0/24/76) & $\begin{array}{c}1,590 / 1,708 / 330 / 123 \\
(42 / 46 / 8 / 3)\end{array}$ & $<0.001$ & $57 / 57 / 13 / 2(44 / 44 / 10 / 2)$ & $<0.001$ \\
\hline ALBI grade (1/2/3), n (\%) & $14 / 21 / 8(33 / 49 / 18)$ & $1,430 / 1,949 / 372(38 / 52 / 10)$ & 0.062 & $58 / 60 / 11(45 / 47 / 8)$ & 0.053 \\
\hline $\begin{array}{l}\text { Performance status } \\
(0 / 1 / 2 / 3-4), n(\%)\end{array}$ & 18/10/9/6 (51/17/15/14) & $\begin{array}{c}2,208 / 770 / 422 / 351 \\
(60 / 21 / 11 / 10)\end{array}$ & 0.018 & $\begin{array}{l}85 / 20 / 14 / 10 \\
(66 / 15 / 11 / 8)\end{array}$ & 0.017 \\
\hline BCLC (0/A/B/C/D), n (\%) & 2/10/3/21/7 (5/23/7/49/16) & $\begin{array}{c}293 / 922 / 637 / 1,483 / 416 \\
(8 / 25 / 17 / 40 / 10)\end{array}$ & 0.282 & $\begin{array}{c}15 / 32 / 24 / 47 / 11 \\
(12 / 28 / 1 / 36 / 9)\end{array}$ & 0.112 \\
\hline
\end{tabular}

Table 1 (continued) 
Table 1 (continued)

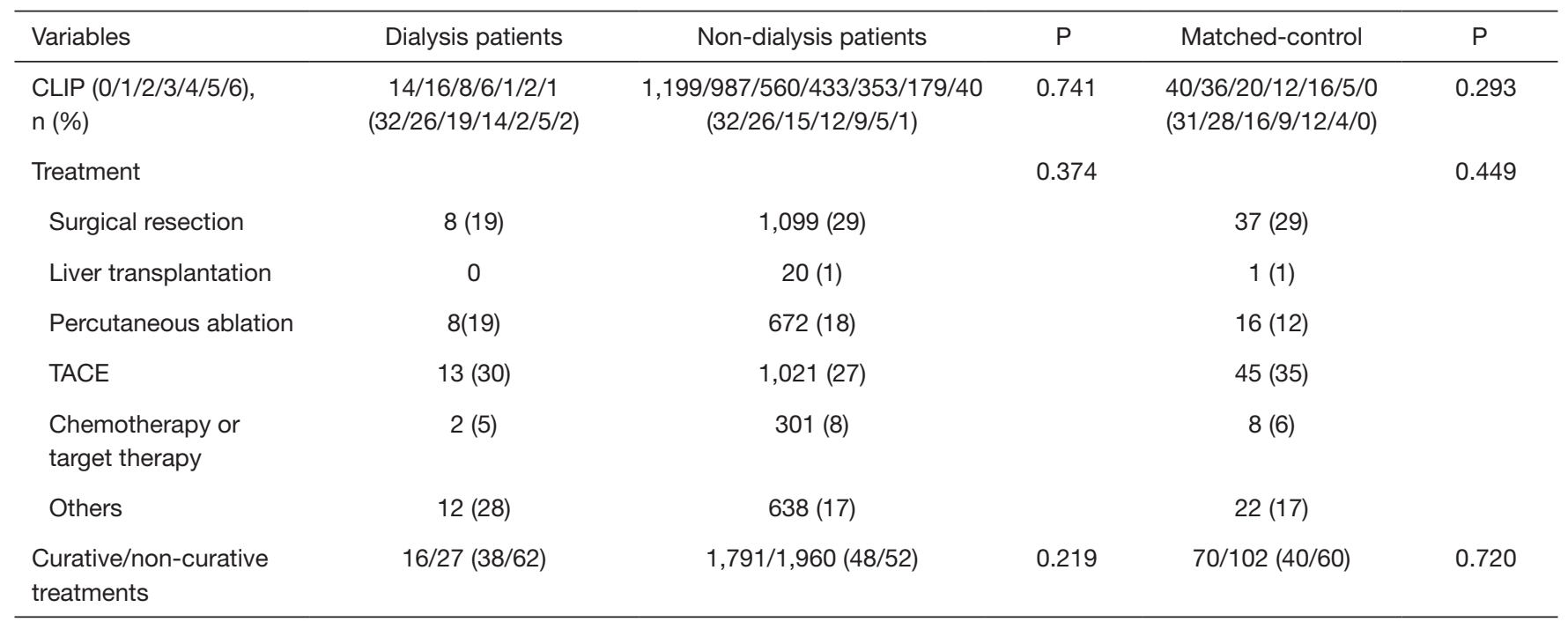

ALBI, albumin-bilirubin; ALT, alanine aminotransferase; AST, aspartate aminotransferase; AFP, a-fetoprotein; CTP, Child-Turcotte-Pugh; DM, diabetes mellitus; INR of PT, international normalized ration of prothrombin time; MELD, model of end-stage liver disease; HBV, hepatitis B virus; HCV, hepatitis C virus; TACE, transarterial chemoembolization; SD, standard deviation.

A

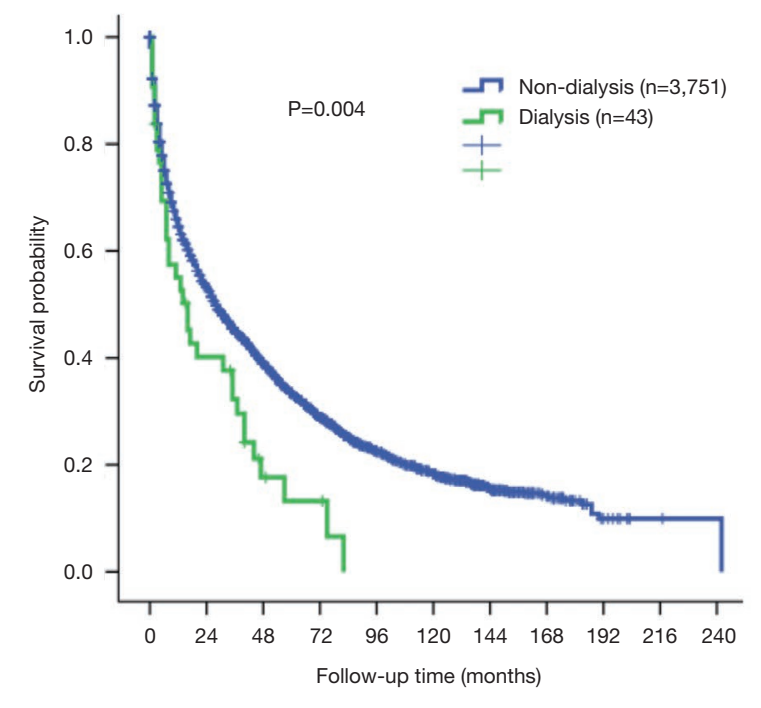

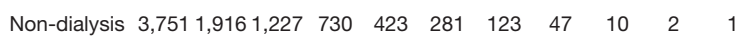

Dialysis $43 \quad 16 \quad 5 \quad 3$

\section{B}

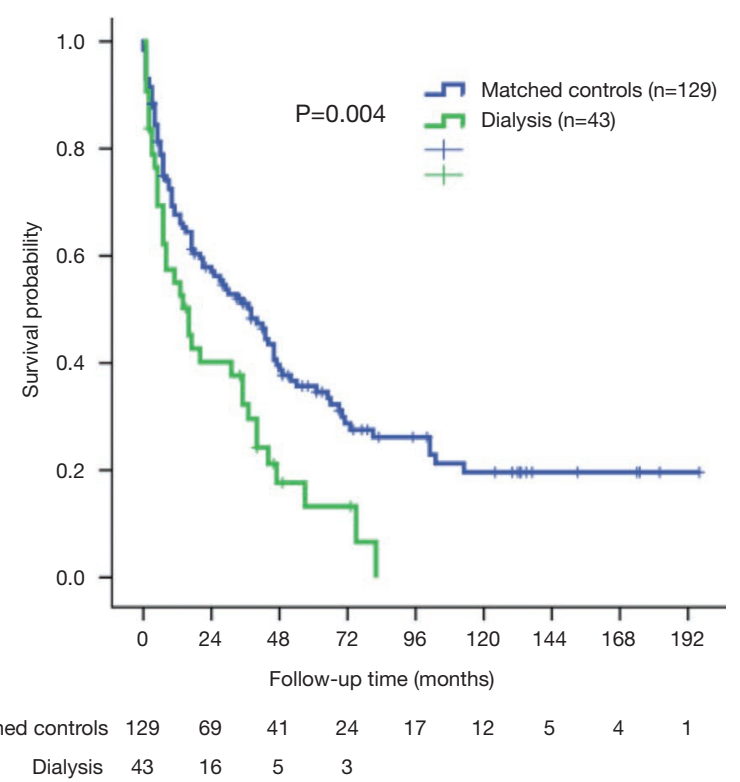

Figure 1 Comparison of survival distribution (A) between dialysis patients and non-dialysis patients with HCC [dialysis patients had a worse survival than non-dialysis patients $(\mathrm{P}=0.004)$ ] and $(\mathrm{B})$ between dialysis patients and age, sex matched controls [dialysis patients still had a worse survival $(\mathrm{P}=0.004)]$. 


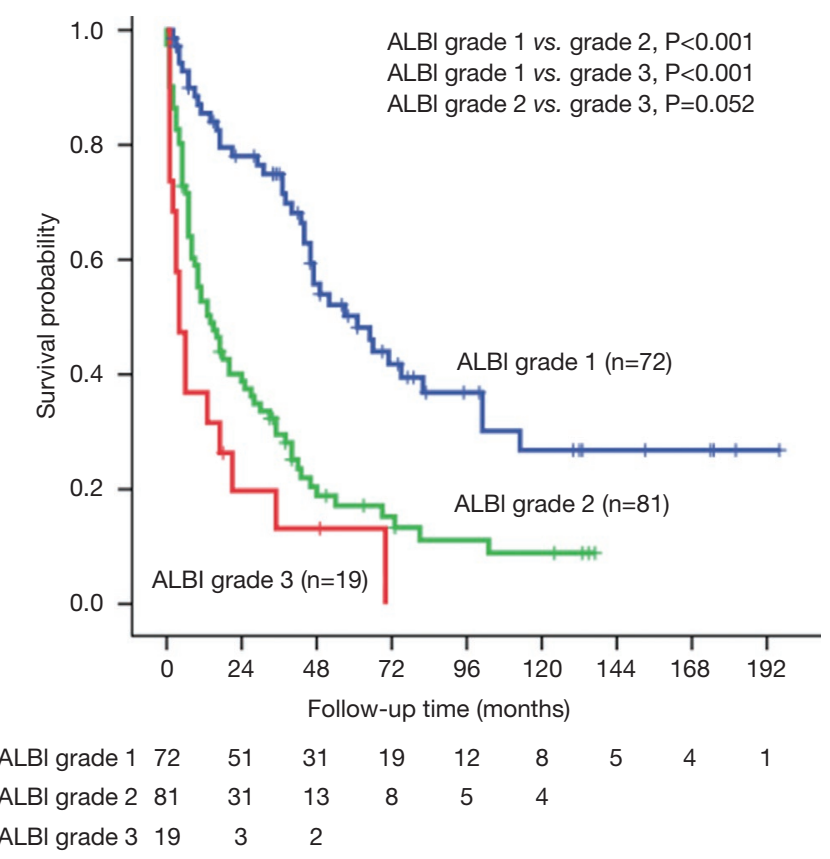

Figure 2 Survival distribution of dialysis and age, sex matched controls stratified by the ALBI grade. ALBI grade 1 patients had the best survival in comparison with other two groups $(\mathrm{P}<0.001)$. ALBI, albumin-bilirubin.

\section{Long-term survival of dialysis patients and matched controls stratified by ALBI grade}

For the 172 (dialysis and matched controls) patients, the median survival was 61 (95\% CI: 40.4-81.6) months for ALBI grade 1, 14 (95\% CI: 7.2-20.8) months for ALBI grade 2, and 4 (95\% CI: 0.8-7.2) months for ALBI grade 3 (Figure 2). There was significant survival difference between ALBI grade 1 vs. grade $2(\mathrm{P}<0.001)$, ALBI grade $1 v s$. grade 3 $(\mathrm{P}<0.001)$, but not between ALBI grade 2 vs. grade 3 $(\mathrm{P}=0.052)$. The 1 -, 3 - and 5 -year survival rates were $86 \%$, $75 \%$ and $50 \%$ for ALBI grade $1,53 \%, 29 \%$ and $17 \%$ for ALBI grade 2 , and $11 \%, 8 \%$ and $0 \%$ for ALBI grade 3 patients, respectively (Figure 2).

\section{Long-term survival of dialysis patients stratified by ALBI grade}

The median survival was 44 (95\% CI: 34.2-53.7) months for ALBI grade 1, 8 (95\% CI: 2-14) months for grade 2, and 1 month for grade 3 patients. There was significant survival difference between ALBI grade 1 vs. grade $2(\mathrm{P}=0.002)$ and ALBI grade $1 v s$. grade $3(\mathrm{P}=0.008)$, but not between ALBI grade 2 vs. grade $3(\mathrm{P}=0.317)$ patients. The 1-, 3 - and 5-year survival rates were $93 \%, 76 \%, 24 \%$ for ALBI grade 1, 43\%, $14 \%$ and $9 \%$ for grade 2 , and $25 \%, 13 \%$ and $0 \%$ for grade 3 patients, respectively (Figure 3).

\section{Univariate and multivariate survival analysis of dialysis patients and matched controls}

In univariate analysis of the pooled cohort of dialysis patients and matched controls, lower serum albumin level $(\mathrm{P}<0.001)$, higher serum bilirubin level $(\mathrm{P}=0.027)$, higher serum ALT level $(\mathrm{P}=0.005)$, prolonged INR of PT $(\mathrm{P}<0.001)$, higher AFP level $(\mathrm{P}<0.001)$, larger TTV $(\mathrm{P}<0.001)$, vascular invasion $(\mathrm{P}<0.001)$, distant metastasis $(\mathrm{P}<0.001)$, ascites $(\mathrm{P}<0.001)$, receiving dialysis $(\mathrm{P}<0.001)$, poor performance status $(\mathrm{P}<0.001)$, higher ALBI grade $(\mathrm{P}<0.001)$ and non-curative treatments $(\mathrm{P}<0.001)$ were associated with an increased risk of mortality.

Multivariate Cox proportional hazards model was used to identify the independent prognostic factors. Serum albumin level and serum bilirubin were not analyzed in the multivariate analysis due to inter-related with ALBI grade. Multivariate analysis revealed that TTV $>33 \mathrm{~cm}^{3}$ (HR: 1.677, 95\% CI: $1.100-2.557, \mathrm{P}=0.016)$, distant metastasis (HR: 2.673, 95\% CI: 1.320-5.412, $\mathrm{P}=0.006$ ), ascites (HR: 1.675 , 95\% CI: $1.106-2.534, \mathrm{P}=0.015$ ), dialysis (HR: 1.751, 95\% CI: $1.165-2.630, \mathrm{P}=0.007$ ), ALBI grade 2 (HR: 2.001, 95\% CI: $1.363-3.164, \mathrm{P}=0.001$ ), ALBI grade 3 (HR: 1.962, 95\% CI: $1.054-3.652, \mathrm{P}=0.034)$, and non-curative treatments (HR: $2.416,95 \%$ CI: $1.554-3.755, \mathrm{P}<0.001$ ) were associated with increased risk of mortality in this pooled cohort (Table 2).

\section{Multivariate survival analysis of dialysis patients}

Of the 43 patients receiving maintenance dialysis, 42 received hemodialysis and 1 received peritoneal dialysis. The median duration of dialysis before the diagnosis of HCC was 36 months (range, 3-167 months). Among dialysis patients, 13 (30\%) of patients had diabetes-related chronic kidney disease. Significant survival differences were found for lower serum albumin level $(\mathrm{P}<0.001)$, higher serum bilirubin level $(\mathrm{P}=0.020)$, higher serum ALT level $(\mathrm{P}=0.024)$, prolonged INR of $\mathrm{PT}(\mathrm{P}=0.004)$, larger TTV $(\mathrm{P}=0.019)$, vascular invasion $(\mathrm{P}<0.001)$, distant metastasis $(\mathrm{P}<0.001)$, ascites $(\mathrm{P}<0.001)$, shorter duration of hemodialysis before the diagnosis of HCC $(\mathrm{P}=0.03)$, diabetes-related cause of dialysis $(\mathrm{P}=0.012)$, performance 
A

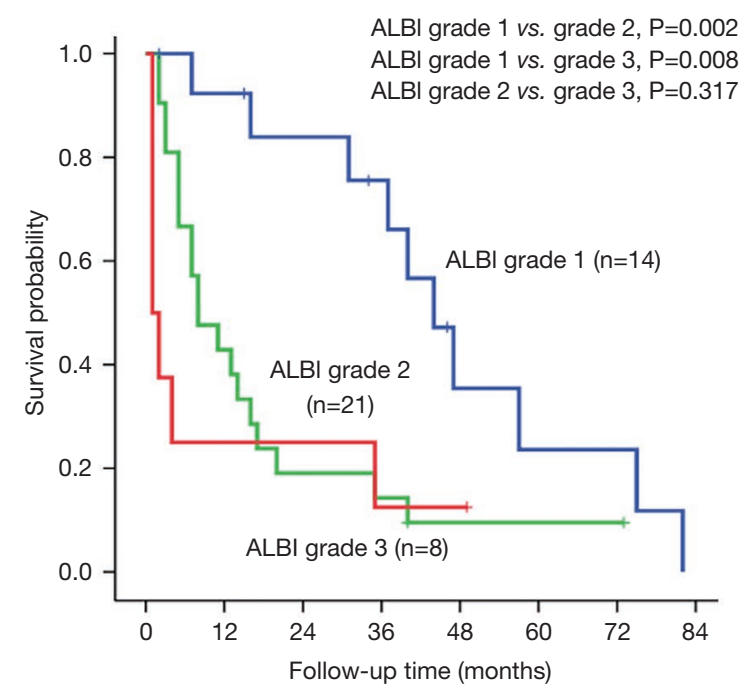

B

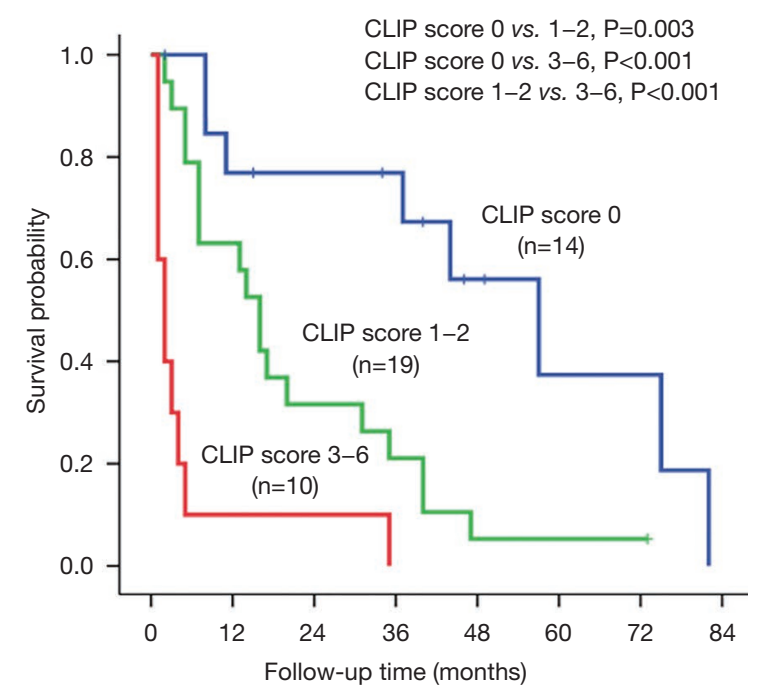

$\begin{array}{rcccccccc}\text { CLIP score 0 } & 14 & 10 & 9 & 8 & 4 & 2 & 2 & 0 \\ \text { CLIP score 1-2 } & 19 & 12 & 6 & 4 & 1 & 1 & 1 & \\ \text { CLIP score 3-6 } & 10 & 1 & 1 & & & & \end{array}$

Figure 3 Survival stratification by ALBI grade and CLIP score. (A) Survival distribution of dialysis patients with HCC stratified by the ALBI grade. ALBI grade 1 patients had the best survival in comparison with other two groups. (B) Survival distribution of dialysis patients with HCC to the CLIP score. There was significant survival difference between CLIP score 0 vs. scores $1-2$ ( $\mathrm{P}=0.03$ ), score 0 vs. scores $3-6$ $(\mathrm{P}<0.001)$, and scores $1-2$ vs. scores 3-6 $(\mathrm{P}<0.001)$. ALBI, albumin-bilirubin.

status $1(\mathrm{P}=0.022)$, performance status 2-3 $(\mathrm{P}=0.004)$, ALBI grade $2(\mathrm{P}=0.005)$, ALBI grade $3(\mathrm{P}<0.001)$ and noncurative treatments $(\mathrm{P}<0.001)$ in the univariate analysis.

The Cox multivariate analysis revealed TTV $>33 \mathrm{~cm}^{3}$ (HR: 6.763, 95\% CI: 2.503-18.270, $\mathrm{P}<0.001$ ), ascites (HR: 6.168, 95\% CI: $2.371-16.403, \mathrm{P}<0.001)$, dialysis duration less than 24 months before diagnosis (HR: 3.144, 95\% CI: 1.388-7.122, $\mathrm{P}=0.006)$, diabetes-related cause of dialysis (HR: 9.366, 95\% CI: 3.320-26.424, $\mathrm{P}=0.001$ ) and noncurative treatments (HR: 9.220, 95\% CI: 3.090-27.510, $\mathrm{P}<0.001)$ as poor prognostic factors of adverse outcome in dialysis patients (Table 3).

\section{Overall survival analysis according to ALBI grade}

There was no overall survival difference between dialysis and non-dialysis patients stratified for ALBI grade 1, 2 and 3 (Figure 4, all $\mathrm{P}>0.05$ ).

\section{Performance of HCC staging systems for dialysis patients}

Among the currently proposed HCC staging systems, the CLIP system offered the lowest AICc value and the highest homogeneity, suggesting that CLIP may better discriminate survival in dialysis patients (Table 4). Further analysis showed that there was significant survival difference between CLIP score 0 vs. scores $1-2(\mathrm{P}=0.003)$, score 0 vs. scores 3-6 $(\mathrm{P}<0.001)$ and scores $1-2$ vs. scores 3-6 $(\mathrm{P}<0.001)$ (Figure $3 B, \mathrm{P}<0.001)$.

\section{Discussion}

Over the 3,794 HCC patients identified between 2002-2017, only 43 (1.1\%) patients had ESRD receiving maintenance dialysis. We specifically investigated and compared their long-term survival with non-dialysis patients. We found that patients receiving dialysis had a significantly decreased overall survival compared with non-dialysis patients. Notably, the ALBI grade can well discriminate the survival difference in the combined cohort of dialysis patients with age and sex-matched controls. We also demonstrate that the CLIP staging system may provide better prognostic accuracy for this specific patient group in comparison with other currently used staging systems. 
Table 2 Univariate and multivariate survival analysis in 43 dialysis and 129 non-dialysis age, sex matched control patients

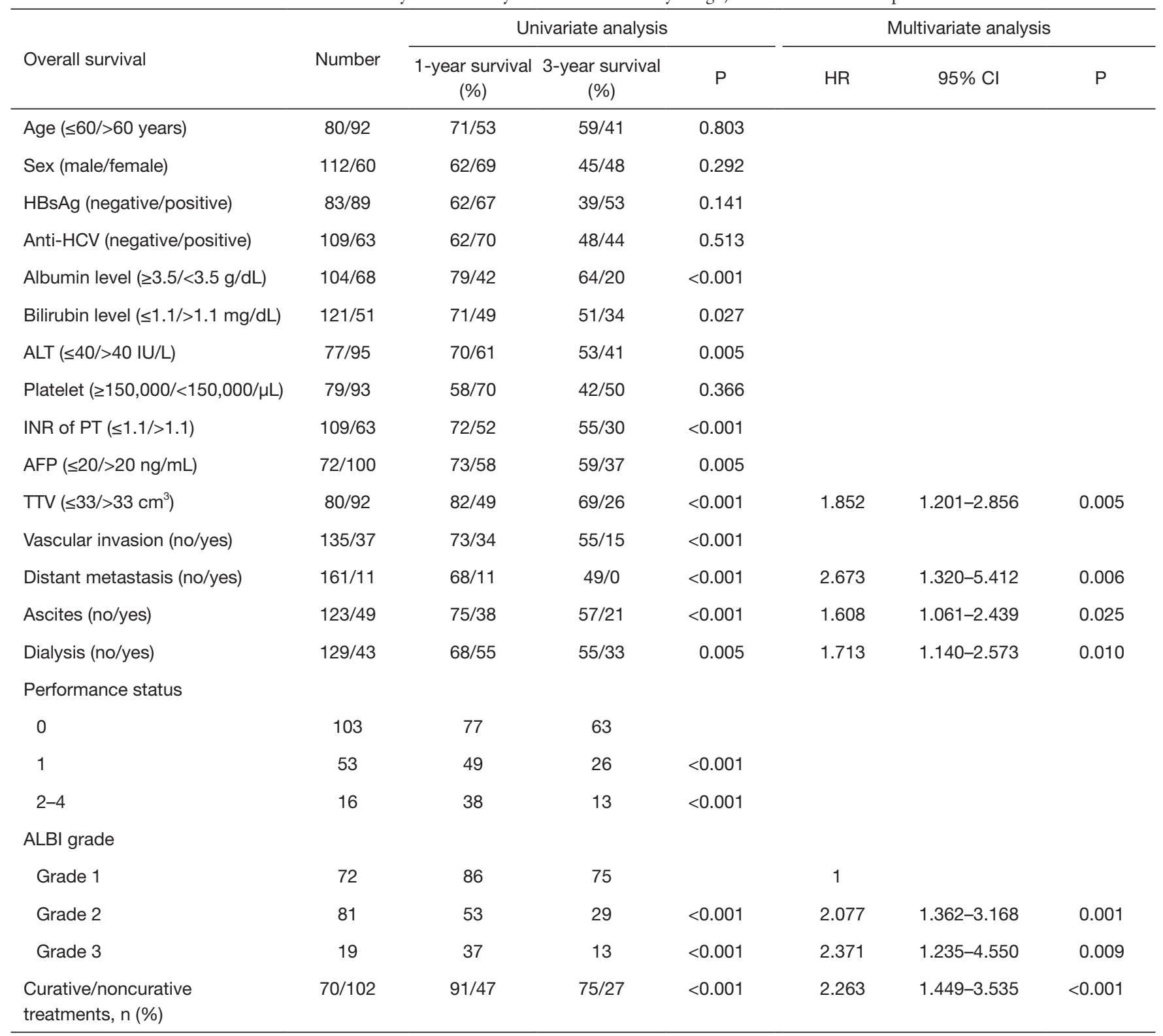

The forepart of the parentheses was set as the reference group in the univariate and multivariate analysis. AFP, a-fetoprotein; ALBI, albumin-bilirubin; ALT, alanine aminotransferase; anti-HCV, antibody against hepatitis C virus; HBsAg, hepatitis B virus surface antigen; INR of PT, international normalized ratio of prothrombin time.

Patients with HCC could develop ESRD during their disease course, but only a minority of these patients were receiving dialysis therapy at the time of diagnosis. Previous studies reported comparable survival rates between dialysis and non-dialysis patients with HCC $(5,24)$. By contrast, another study from Japan revealed that patients receiving dialysis had lower survival rate compared with non-dialysis patients (25). Therefore, the prognosis of HCC patients with dialysis was highly debated. In this study, we confirmed that dialysis patients had a poor long-term survival compared with non-dialysis patients, with $71 \%$ increased risk of mortality identified in the multivariate model.

When compared with non-dialysis patients at baseline, patients receiving dialysis had lower serum albumin level, higher serum bilirubin level and higher rate of ascites formation, indicating poor liver functional reserve. In 
Table 3 Univariate and multivariate survival analysis in dialysis HCC patients $(n=43)$

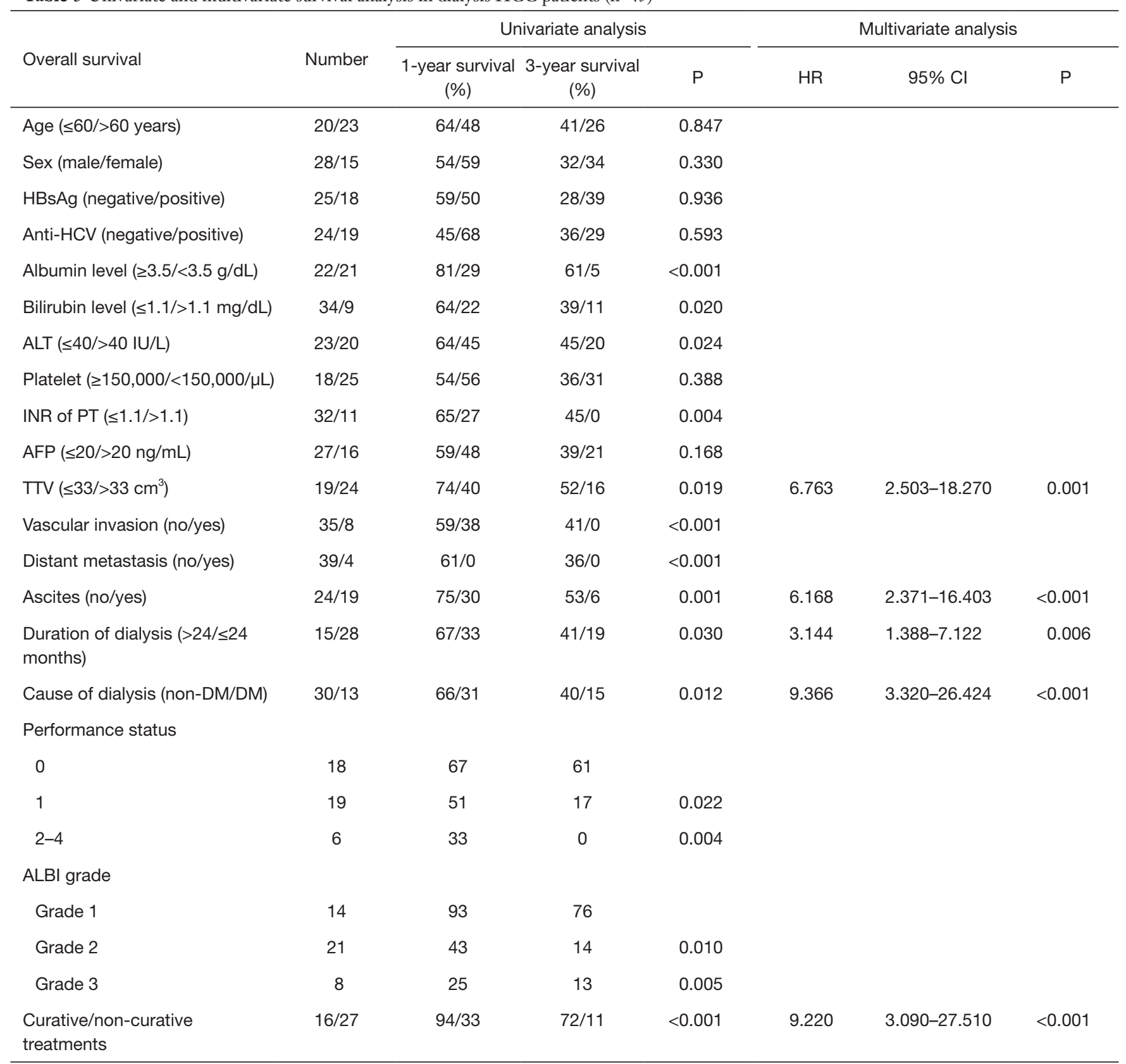

The forepart of the parentheses was set as the reference group in the univariate and multivariate analysis. AFP, a-fetoprotein; ALBI, albumin-bilirubin; ALT, alanine aminotransferase; anti-HCV, antibody against hepatitis C virus; HBsAg, hepatitis B virus surface antigen; INR of PT, international normalized ratio of prothrombin time.

addition, dialysis patients more frequently had poor performance status and advanced cancer stage. These characteristics may decrease the likelihood to receive aggressive treatment associated with unfavorable long-term survival in dialysis patients.

The degree of liver functional reserve is a crucial prognostic predictor in the management of HCC. The CTP classification and the MELD score have been used to predict the outcome in cirrhotic patients. However, about $20 \%$ of HCC did not have cirrhosis at the time of diagnosis. Furthermore, the value of creatinine in the MELD equation was set at $4 \mathrm{mg} / \mathrm{dL}$ in dialysis patients, and this 
A Survival distribution of ALBI grade 1 patients $(n=1,444)$

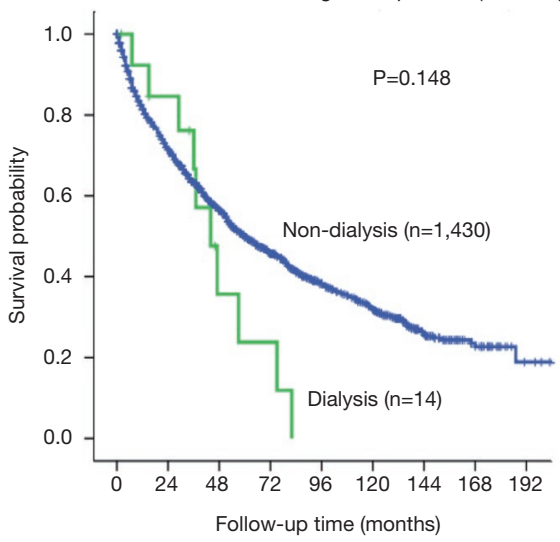

Dialysis $1,430999 \quad 702 \quad 460 \quad 280 \quad 190 \quad 73 \quad 26 \quad 4$ Non-dialysis $\begin{array}{llll}14 & 10 & 3 & 2\end{array}$

B

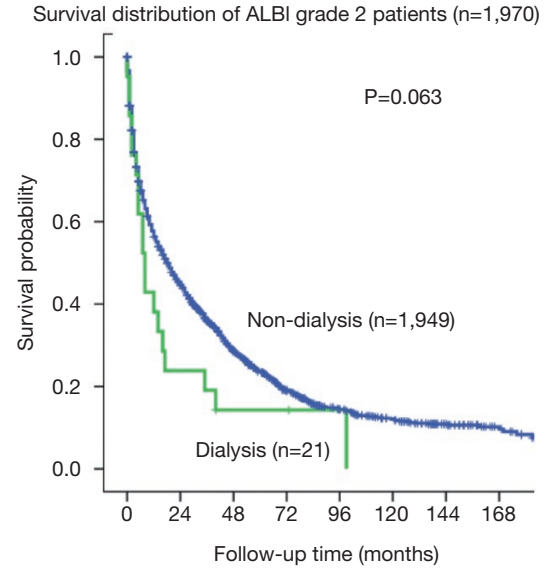

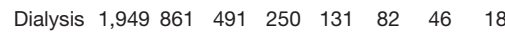
Non-dialysis $\begin{array}{lllll}21 & 4 & 1 & 1\end{array}$

C Survival distribution of ALBI grade 3 patients $(n=380)$

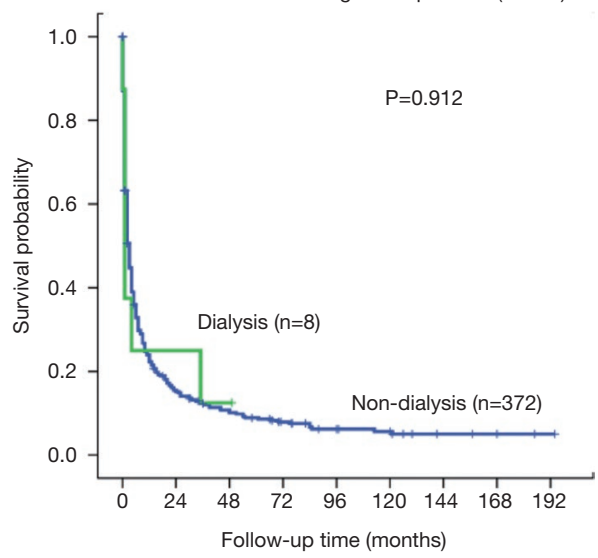

$\begin{array}{llllllllll}\text { Dialysis } & 372 & 56 & 34 & 20 & 12 & 9 & 4 & 3 & 1\end{array}$

Non-dialysis $8 \quad 2$

Figure 4 Comparison of survival between dialysis and non-dialysis patients according to ALBI grade. There were no significant survival differences between dialysis and non-dialysis patients stratified for ALBI grade 1 (A), grade 2 (B) and grade 3 (C).
Table 4 Prognostic performance of different staging systems in dialysis patients with HCC $(\mathrm{n}=43)$

\begin{tabular}{lcc}
\hline Staging system & Homogeneity $\left(\right.$ Wald $\left.\chi^{2}\right)$ & AlCc \\
\hline BCLC & 18.712 & 195.407 \\
CLIP & 32.025 & 182.095 \\
CUPI & 2.464 & 192.310 \\
HKLC & 20.790 & 183.897 \\
JIS & 25.704 & 188.416 \\
Okuda & 9.086 & 205.033 \\
TNM & 8.139 & 205.981 \\
Tokyo & 20.930 & 193.190 \\
TIS & 17.388 & 196.731
\end{tabular}

BCLC, Barcelona Clinic of Liver Cancer; CLIP, Cancer of the Liver Italian Program; CUPI, Chinese University Prognostic Index; HKLC, Hong Kong Liver Cancer; JIS, Japan Integrated Scoring; TNM, tumor-node-metastasis; TIS, Taipei Integrated Scoring system.

score is considered less reliable in these patients. Therefore, the predictive accuracy of these two models to assess liver dysfunction in chronic liver disease or mild cirrhosis has been challenged. More recently, the application of ALBI grade to evaluate liver functions in HCC was proposed, but its prognostic role in dialysis patients with HCC is unclear. Our results confirm that patients with ALBI grade can well discriminate survival difference among HCC patients with dialysis and matched controls. Notably, patients with ALBI grade 2 and grade 3 had 2.1- and 2.4-fold increased risk of mortality, respectively, compared with patients ALBI grade 1 in multivariate analysis. Alternatively, in the analysis for solely dialysis patients, ALBI grade was not an independent prognostic predictor. Rather, tumor burden, treatment strategy, and the cause of dialysis prevailed and determined the survival of these patients.

Among the cohort of dialysis and age, sex-matched controls, TTV, distant metastasis, ascites, patients receiving dialysis, ALBI grade, and treatment strategy were identified as independent predictors of poor survival. We also evaluated the prognostic determinants in solely dialysis patients with HCC and found that larger TTV, ascites, duration of dialysis $\leq 24$ months, diabetes-related cause of dialysis, and non-curative treatments were associated with decreased long-term survival. These results were largely consistent with previous studies (24-26). Notably, those with shorter duration of dialysis were associated with poor 
outcome. The possible explanation is that the development of ESRD may be directly related to advanced cirrhosis resulting in decreased survival in these patients. The cause of dialysis related to DM is another predictor of poor outcome compared with those with non-diabetes related dialysis. The exact mechanism remains unclear; however, in accordance with previous studies, DM was regarded as a predictor of poor survival in dialysis HCC patients (26-28).

Ascites formation is a hallmark of portal hypertension. Previous study revealed that the presence of ascites was not only related to advanced cirrhosis but may also predispose to tumor progression. Our data consistently showed that ascites was associated with 6.2-fold increased risk of death compared with those without ascites $(7,29)$.

Treatment strategy is usually the single most important factor to predict survival for HCC (2). Patients receiving curative treatments had significantly better 1 - and 3-year survival compared with those undergoing non-curative treatments in the cohort of dialysis patients and matched controls. In this study, about $38 \%$ of dialysis patients with HCC received curative treatments which were associated with significantly improved outcome. These results imply that curative treatments can be safely performed in dialysis patients with well-preserved liver function and good performance status (30), and to improve overall survival in HCC patients receiving dialysis.

Dialysis patients are associated with increased risk of $\mathrm{HBV}$ and $\mathrm{HCV}$ infection transmitted primarily through the dialysis environment. A previous study reported that hemodialysis patients had at higher risk of early HCV infection which may in turn progress to liver cirrhosis and HCC (31). Consistent with this finding, our results showed that $\mathrm{HCV}$ infection was more common in dialysis patients compared with non-dialysis patients. Additionally, patients receiving hemodialysis are characterized by a higher prevalence of dual HBV/HCV infection, suggesting the importance of virus screening in these patients.

Multiple staging systems have been suggested for HCC. However, the best staging system specifically for dialysis patients with $\mathrm{HCC}$ is undetermined. Our findings suggest that the CLIP score, which had the lowest AICc and highest homogeneity compared with other staging systems, had a better prognostic performance to discriminate survival in dialysis patient with HCC.

Liver dysfunction in the setting of liver cirrhosis or HCC is associated with high mortality due to the accumulation of protein-bound metabolites, such as bilirubin which is not removed by conventional hemodialysis. Alternatively, albumin levels are lower in dialysis patients than among the general population and are a powerful predictor of mortality. Albumin levels are mainly controlled by the rate of albumin synthesis that is in turn affected by the nutritional status. In dialysis patients, hypoalbuminemia is a strong predictor of poor outcome (32). Given the fact that dialysis itself could not alter these levels, the clinical impact of dialysis in terms of prognostic prediction is probably in multiple ways. Still, our results suggest that ALBI grade can serve as a prognostic marker in dialysis patients.

This study has some potential shortcomings. First, this is a single-center study from Asian-Pacific region. $\mathrm{HBV}$ is the predominant etiology of HCC and the results require external validation from other search groups where different etiologies prevail. Second, the vast majority of dialysis patients received hemodialysis and only one patient received peritoneal dialysis. The assessment between hemodialysis and peritoneal dialysis would be difficult. Third, some patients did not fully comply with treatment recommendations according to the BCLC system. The anti-cancer treatments were mainly decided by the multidisciplinary HCC board in our hospital. Lastly, dialysis patients are a heterogeneous group that could contain inherent biases in comparison with other patient groups. Although the Cox multivariate analysis is used to adjust the confounders, there still could be potentially uncontrolled factors in data interpretation.

\section{Conclusions}

In conclusions, dialysis patients with HCC had decreased overall survival compared with non-dialysis patients. The duration of hemodialysis, ascites, tumor burden, distant metastasis, and treatment strategy were associated with long-term outcome of HCC patients receiving dialysis. The ALBI grade is a feasible model to evaluate the severity of liver injury in dialysis patients with HCC, and the CLIP score can better stage their long-term prognosis.

\section{Acknowledgments}

Funding: This study was supported by the grants from Taipei Veterans General Hospital, Taipei Taiwan (V109C-011, VN109-06, V108C-008).

\section{Footnote}

Reporting Checklist: The authors have completed the 
REMARK reporting checklist. Available at http://dx.doi. org/10.21037/jgo-20-332

Data Sharing Statement: Available at http://dx.doi. org/10.21037/jgo-20-332

Conflicts of Interest: All authors have completed the ICMJE uniform disclosure form (available at http://dx.doi. org/10.21037/jgo-20-332). The authors have no conflicts of interest to declare.

Ethical Statement: The authors are accountable for all aspects of the work in ensuring that questions related to the accuracy or integrity of any part of the work are appropriately investigated and resolved. The study was approved by the Institutional Review Board of Taipei Veterans General Hospital (No. 2018-03-004CC) and complies with the standards of the Declaration of Helsinki (as revised in 2013) and current ethical guidelines. Informed consent was obtained before treatment.

Open Access Statement: This is an Open Access article distributed in accordance with the Creative Commons Attribution-NonCommercial-NoDerivs 4.0 International License (CC BY-NC-ND 4.0), which permits the noncommercial replication and distribution of the article with the strict proviso that no changes or edits are made and the original work is properly cited (including links to both the formal publication through the relevant DOI and the license). See: https://creativecommons.org/licenses/by-nc-nd/4.0/.

\section{References}

1. Villanueva A. Hepatocellular Carcinoma. N Engl J Med 2019;380:1450-62.

2. EASL Clinical Practice Guidelines: Management of hepatocellular carcinoma. J Hepatol 2018;69:182-236.

3. Frakes JM, Abuodeh YA, Naghavi AO, et al. Viral hepatitis associated hepatocellular carcinoma outcomes with yttrium-90 radioembolization. J Gastrointest Oncol 2018;9:546-52.

4. Ginès $P$, Schrier RW. Renal failure in cirrhosis. N Engl J Med 2009;361:1279-90.

5. Chen CF, Liu PH, Lee YH, et al. Impact of renal insufficiency on patients with hepatocellular carcinoma undergoing radiofrequency ablation. J Gastroenterol Hepatol 2015;30:192-8.

6. Orii T, Takayama T, Haga I, et al. Efficacy of a liver resection for hepatocellular carcinoma in patients with chronic renal failure. Surg Today 2008;38:329-34.

7. Hsu CY, Huang $\mathrm{YH}, \mathrm{Su} \mathrm{CW}$, et al. Renal failure in patients with hepatocellular carcinoma and ascites undergoing transarterial chemoembolization. Liver Int 2010;30:77-84.

8. Go AS, Chertow GM, Fan D, et al. Chronic kidney disease and the risks of death, cardiovascular events, and hospitalization. N Engl J Med 2004;351:1296-305.

9. Aghakhani A, Banifazl M, Kalantar E, et al. Occult hepatitis $B$ virus infection in hemodialysis patients with isolated hepatitis B core antibody: a multicenter study. Ther Apher Dial 2010;14:349-53.

10. Wigneswaran J, Van Wyck D, Pegues D, et al. Hepatitis $\mathrm{C}$ virus infection in patients with end-stage renal disease. Hemodial Int 2018;22:297-307.

11. Wiesner R, Edwards E, Freeman R, et al. Model for endstage liver disease (MELD) and allocation of donor livers. Gastroenterology 2003;124:91-6.

12. Johnson PJ, Berhane S, Kagebayashi C, et al. Assessment of liver function in patients with hepatocellular carcinoma: a new evidence-based approach-the ALBI grade. J Clin Oncol 2015;33:550-8.

13. Huo TI. ALBI grade as a new player in hepatocellular carcinoma. J Chin Med Assoc 2019;82:1.

14. Ho SY, Hsu CY, Liu PH, et al. Albumin-bilirubin gradebased nomogram of the BCLC system for personalized prognostic prediction in hepatocellular carcinoma. Liver Int 2020;40:205-14.

15. Mohammadi H, Abuodeh $\mathrm{Y}$, Jin W, et al. Using the Albumin-Bilirubin (ALBI) grade as a prognostic marker for radioembolization of hepatocellular carcinoma. J Gastrointest Oncol 2018;9:840-6.

16. Liu PH, Hsu CY, Hsia CY, et al. Prognosis of hepatocellular carcinoma: Assessment of eleven staging systems. J Hepatol 2016;64:601-8.

17. Shah C, Mramba LK, Bishnoi R, et al. Survival differences among patients with hepatocellular carcinoma based on the stage of disease and therapy received: pre and post sorafenib era. J Gastrointest Oncol 2017;8:789-98.

18. Hsu CY, Liu PH, Ho SY, et al. Metastasis in patients with hepatocellular carcinoma: Prevalence, determinants, prognostic impact and ability to improve the Barcelona Clinic Liver Cancer system. Liver Int 2018;38:1803-11.

19. Hsu CY, Lee YH, Hsia CY, et al. Performance status in patients with hepatocellular carcinoma: determinants, prognostic impact, and ability to improve the Barcelona Clinic Liver Cancer system. Hepatology 2013;57:112-9. 20. Hsu CY, Huang YH, Hsia CY, et al. A new prognostic 
model for hepatocellular carcinoma based on total tumor volume: the Taipei Integrated Scoring System. J Hepatol 2010;53:108-17.

21. Huo TI, Wang YW, Yang YY, et al. Model for endstage liver disease score to serum sodium ratio index as a prognostic predictor and its correlation with portal pressure in patients with liver cirrhosis. Liver Int 2007;27:498-506.

22. Levey AS, Coresh J, Greene T, et al. Using standardized serum creatinine values in the modification of diet in renal disease study equation for estimating glomerular filtration rate. Ann Intern Med 2006;145:247-54.

23. Hsu CY, Liu PH, Hsia CY, et al. Surgical Resection is Better than Transarterial Chemoembolization for Patients with Hepatocellular Carcinoma Beyond the Milan Criteria: A Prognostic Nomogram Study. Ann Surg Oncol 2016;23:994-1002.

24. Lee YH, Hsu CY, Hsia CY, et al. Hepatocellular carcinoma in uremic patients: is there evidence for an increased risk of mortality? J Gastroenterol Hepatol 2013;28:348-56.

25. Toyoda H, Hiraoka A, Tada T, et al. Characteristics and Prognosis of Hepatocellular Carcinoma in Japanese Patients Undergoing Dialysis. Ther Apher Dial 2017;21:465-72.

26. Huo TI, Hsu CY, Huang YH, et al. Diabetes mellitus as an independent prognostic predictor and its association

Cite this article as: Ho SY, Liu PH, Hsu CY, Ko CC, Huang YH, Su CW, Hsia CY, Lee RC, Hou MC, Huo TI. ALBI grade in dialysis patients with hepatocellular carcinoma: prognostic impact and staging strategy. J Gastrointest Oncol 2021;12(2):722-734. doi: 10.21037/jgo-20-332 with renal dysfunction in patients with hepatocellular carcinoma. Liver Int 2010;30:198-207.

27. Li Z, Gao Z, Xiang J, et al. Intention-to-treat analysis of liver transplantation for hepatocellular carcinoma: The impact of pre-existing diabetes mellitus. Liver Int 2019;39:361-70.

28. Huo TI, Wu JC, Lui WY, et al. Diabetes mellitus is a recurrence-independent risk factor in patients with hepatitis B virus-related hepatocellular carcinoma undergoing resection. Eur J Gastroenterol Hepatol 2003;15:1203-8.

29. EASL clinical practice guidelines on the management of ascites, spontaneous bacterial peritonitis, and hepatorenal syndrome in cirrhosis. J Hepatol 2010;53:397-417.

30. Cheng SB, Wu CC, Shu KH, et al. Liver resection for hepatocellular carcinoma in patients with end-stage renal failure. J Surg Oncol 2001;78:241-6; discussion 246-7.

31. Johnson DW, Dent H, Yao Q, et al. Frequencies of hepatitis $\mathrm{B}$ and $\mathrm{C}$ infections among haemodialysis and peritoneal dialysis patients in Asia-Pacific countries: analysis of registry data. Nephrol Dial Transplant 2009;24:1598-603.

32. Kaysen GA. Serum albumin concentration in dialysis patients: why does it remain resistant to therapy? Kidney Int Suppl 2003;87:S92-8. 